\title{
Intracellular Glutathione in the Protection from Anoxic Injury in Renal Proximal Tubules
}

\author{
Lazaro J. Mandel, Rick G. Schnellmann, and William R. Jacobs \\ With the technical assistance of Maria Sgambati, Gema Gomez, Heidi Adamek, and Kolette Fly \\ Machu Picchu Research Foundation, Division of Physiology, Department of Cell Biology,
}

Duke University Medical Center, Durham, North Carolina 27710

\begin{abstract}
Previous results (Weinberg, J. M., J. A. David, M. Abarzua, and T. Rajan. 1987. J. Clin. Invest. 80:1446-1454) have shown that GSH and glycine (GLY) are cytoprotective during anoxia when added extracellularly. The present studies investigate the role that intracellular GSH plays in this cytoprotection. Proximal renal tubules in suspension prepared with either high (11 $\pm 1 \mathrm{nmol} / \mathrm{mg}$ protein) or low (6 $\pm 1 \mathrm{nmol} / \mathrm{mg}$ protein) $\mathrm{GSH}$ contents were subjected to $40 \mathrm{~min}$ of anoxia and $40 \mathrm{~min}$ of reoxygenation. Low GSH tubules were protected from plasma membrane damage during anoxia by exogenous addition of 1 mM GSH or GLY, reducing lactate dehydrogenase (LDH) release from $42 \pm 7$ to $14 \pm 1$ and $10 \pm 1 \%$, respectively. High GSH tubules were equally protected from anoxic damage without exogenous additions. Since the high GSH content approximates the in vivo values, it may be concluded that GSH may be cytoprotective during anoxia in vivo. However, it is not the intracellular GSH itself that is cytoprotective; rather, this protection resides in the ability to produce GLY, which appears to be the cytoprotective agent. Alanine was also shown to have similar cytoprotective properties, although higher concentrations were required. Sulfhydryl reducing agents such as cysteine and dithiothreitol offered less, but significant protection from anoxic damage. Protection by GSH, GLY, or alanine was not associated with higher ATP levels during anoxia. Tubules that were protected from membrane damage during anoxia recovered oxygen consumption and $K$ and ATP contents significantly better during reoxygenation than unprotected tubules. (J. Clin. Invest. 1990. 85:316-324.) alanine - cysteine • glycine $\bullet$ ischemia $\bullet$ rabbit
\end{abstract}

\section{Introduction}

GSH is found in numerous tissues, including the kidney, in concentrations of $0.1-10 \mathrm{mM}(1,2)$. This compound seems to

Dr. Schnellmann's present address is Department of Physiology and Pharmacology, College of Veterinary Medicine, University of Georgia, Athens, GA 30602. Dr. Jacobs' present address is Department of Pediatrics, University of Tennessee Medical Center at Knoxville, 1924 Alcoa Highway, Knoxville, TN 37920-6999.

Address correspondence to Dr. Mandel, Machu Picchu Research Foundation, Division of Physiology, Department of Cell Biology, Duke University Medical Center, Durham, NC 27710.

Received for publication 25 April 1989 and in revised form 29 September 1989.

J. Clin. Invest.

(C) The American Society for Clinical Investigation, Inc.

0021-9738/90/02/0316/09 \$2.00

Volume 85, February 1990, 316-324 be involved in a number of cellular reactions, including the detoxification of reactive oxygen species $(1,2)$. Various studies performed in vivo have found a protective role for GSH when either the liver or the kidney was subjected to an ischemic episode followed by reperfusion (3-5). This protection has been attributed to the ability of GSH to reduce oxygen free radicals through GSH peroxidase. Recent experiments using GSH supplementation achieved mixed results, one study finding significant protection (6), while another showed no protective effect (7). Although these experiments are provocative, they do not allow a clear characterization of the site or time of protective action, whether it was within the vascular tissue or in the epithelial cells, or whether it occurred during anoxia or reoxygenation.

The proximal tubule suspension is a model system in which effects on the tubular epithelium can be separated from vascular endothelial effects and hemodynamic considerations. Our previous studies in rabbit tubules show that $40 \mathrm{~min}$ of anoxia produce irreversible damage, with no further damage occurring during reoxygenation $(8,9)$. Furthermore, we found that prevention of plasma membrane damage during anoxia led to complete recovery after $40 \mathrm{~min}$ of reoxygenation. A recent report by Weinberg et al. (10) demonstrated striking protective effects of GSH or glycine (GLY) ${ }^{1}$ added to the extracellular medium during anoxia. The present study investigates this finding in rabbit tubules further. We specifically examined the role that intracellular GSH plays in the aforementioned protection, since this variable was not measured in the previous study (10). In addition, a clear separation was made between protective effects obtained during anoxia from those obtained during reoxygenation. Results show that tubules with high initial GSH content were better protected during anoxia and reoxygenation than tubules with low initial GSH content. However, intracellular GSH itself does not appear to be the protective agent; rather, this protection appears to reside in the ability to produce more GLY, a constituent amino acid of GSH, which appears to be directly cytoprotective, as previously described (10).

\section{Methods}

Preparation of renal tubule suspension. Renal cortical tubules were prepared from female New Zealand White rabbits (3-4 kg wt; Bunny Haven, Durham, NC) according to previously published methods (11, 12). In brief, after the animal was injected with heparin and anesthetized with ether, both kidneys were perfused at $37^{\circ} \mathrm{C}$ with a medium containing $115 \mathrm{mM} \mathrm{NaCl}, 25 \mathrm{mM} \mathrm{NaHCO}_{3}, 2 \mathrm{mM} \mathrm{NaH}_{2} \mathrm{PO}_{4}, 1 \mathrm{mM}$

1. Abbreviations used in this paper: BSO, buthionine sulfoximine; CYS, cysteine; DEM, diethylmaleate; GLY, glycine; HX, hypoxanthine; LDH, lactate dehydrogenase. 
$\mathrm{CaCl}_{2}, 5 \mathrm{mM} \mathrm{KCl}, 1 \mathrm{mM} \mathrm{MgSO}_{4}, 5 \mathrm{mM}$ glucose, $4 \mathrm{mM}$ lactate, $1 \mathrm{mM}$ alanine, $0.6 \%$ dextran, and $25 \mathrm{mM}$ mannitol equilibrated with $95 \%$ $\mathrm{O}_{2} / 5 \% \mathrm{CO}_{2}$ ( $\mathrm{pH}$ 7.4). After clearing the blood from the kidneys with this solution, the perfusion was continued with the same solution to which $124 \mathrm{U} / \mathrm{ml}$ collagenase was added, followed by the original solution to clear the collagenase. The cortex was then excised, minced, dispersed, and washed three times with ice-cold medium with the same original composition except for the mannitol and dextran. To remove nonvital single cells and cellular debris, one of the washes involved the use of a Ficoll cushion. Approximately $90 \%$ of tubules in the resulting preparation were proximal in origin and their lumens were open. The final pellet was resuspended at a concentration of 4-6 $\mathrm{mg}$ protein $/ \mathrm{ml}$ in the incubation medium containing $115 \mathrm{mM} \mathrm{NaCl}, 15 \mathrm{mM} \mathrm{NaHCO}_{3}$, $2 \mathrm{mM} \mathrm{NaH}_{2} \mathrm{PO}_{4}, 1 \mathrm{mM} \mathrm{CaCl}_{2}, 5 \mathrm{mM} \mathrm{KCl}, 1 \mathrm{mM} \mathrm{MgSO}_{4}, 5 \mathrm{mM}$ glucose, $4 \mathrm{mM}$ lactate, $1 \mathrm{mM}$ alanine, $5 \mathrm{mM}$ glutamate, $5 \mathrm{mM}$ malate, $1 \mathrm{mM}$ valerate, $10 \mathrm{mM}$ Hepes ( $\mathrm{pH} 7.4$ ), and $0.6 \%$ dextran, equilibrated with $95 \% \mathrm{O}_{2} / 5 \% \mathrm{CO}_{2}$.

The procedure described above was used in most experiments. It produced tubules with GSH contents of $4-6 \mathrm{nmol} / \mathrm{mg}$ protein, less than half the GSH content of $13 \mathrm{nmol} / \mathrm{mg}$ protein normally found in vivo (13). In some experiments tubules were prepared with GSH contents similar to those found in vivo. To obtain the higher GSH contents, $10 \mathrm{mM}$ GSH was added to all the preparative steps in the cold, followed by a 20 -min preincubation at $37^{\circ} \mathrm{C}$ in the incubation medium to which $1 \mathrm{mM} \mathrm{GSH}$ was added. This procedure was followed by a wash to remove extracellular GSH and its breakdown products and further incubation for $10 \mathrm{~min}$ at $37^{\circ} \mathrm{C}$ before the initiation of anoxia. The GSH contents of these tubules sampled just before anoxia averaged $11 \mathrm{nmol} / \mathrm{mg}$ protein. A comparison was made (see Figs. 1 and 2) between these tubules and a paired group in which the initial GSH content was depleted before anoxia. The depletion was achieved by reducing the $37^{\circ} \mathrm{C}$ preincubation time in $1 \mathrm{mM}$ GSH to $10 \mathrm{~min}$, washing with GSH-free incubation medium to remove extracellular $\mathrm{GSH}$, and a $10-\mathrm{min}$ incubation at $37^{\circ} \mathrm{C}$ in incubation medium to which $2 \mathrm{mM}$ diethylmaleate (DEM) was added to alkylate the intracellular GSH. The DEM was removed by washing, followed by a final 10 -min preincubation at $37^{\circ} \mathrm{C}$ in fresh incubation medium. This procedure reduced the intracellular GSH content to an average of 2.5 $\mathrm{nmol} / \mathrm{mg}$ protein before anoxia.

Anoxia experiments. Each experiment was initiated with a 20-30min preequilibration of the tubule suspension at $37^{\circ} \mathrm{C}$ in the incubation solution bubbled with $95 \% \mathrm{O}_{2} / 5 \% \mathrm{CO}_{2}$. The tubules were then enclosed in a specially designed thermostatted chamber (11) and allowed to consume all the oxygen in the solution. Anoxia was achieved when the $\mathrm{PO}_{2}$ could not be distinguished from zero by the Clark electrode in the chamber. Addition of either $1 \mathrm{mM} \mathrm{MgCl}_{2}$ (control), ${ }^{2}$ or 1 mM GSH, GLY, cysteine (CYS), GLY + buthionine sulfoximine (BSO), CYS + BSO, or DTT were made at this time. This concentration was selected because Weinberg et al. (10) found it to be maximally effective for these compounds. Anoxia was maintained for $40 \mathrm{~min}$. After this period of anoxia the tubule suspension was reoxygenated for $40 \mathrm{~min}$ in a shaker bath under a $95 \% \mathrm{O}_{2} / 5 \% \mathrm{CO}_{2}$ atmosphere at $37^{\circ} \mathrm{C}$. Samples were taken from the suspension three times during each experiment: immediately before anoxia, immediately after anoxia, and after reoxygenation. Previous experiments $(8,9)$ have shown that tubule suspensions were stable under oxygenated conditions for the duration of these experiments. Therefore, percent impairment of each function was calculated using the respective control value obtained just before anoxia. At each sampling time nystatin-stimulated respiration, percentage of lactate dehydrogenase (LDH) released from the cells, total cellular K and GSH contents, and cellular and extracellular con-

2. Control tubules were treated with $1 \mathrm{mM} \mathrm{MgCl}$ to provide the same conditions as in our previous studies (reference 9), in which all nucleotide and nucleoside additions were made with $1 \mathrm{mM} \mathrm{MgCl}$. This addition produced no observable change in the tubules. tents of adenine nucleotides, and their breakdown products were measured.

Nystatin-stimulated respiration. Previous studies in our laboratory (14) have determined that addition of nystatin to proximal tubules leads to a rapid increase in oxygen consumption $\left(\mathrm{QO}_{2}\right)$ caused by the entry of sodium from the extracellular medium and the consequent stimulation of $\mathrm{Na}, \mathrm{K}-\mathrm{ATPase}$ activity. The $\mathrm{QO}_{2}$ obtained with nystatin addition has been shown to equal the mitochondrial state 3 respiratory rate under normal conditions (14). Therefore, nystatin-stimulated $\mathrm{QO}_{2}$ was used to measure the maximal rate of coupled respiration obtainable in the intact tubules before and after anoxia or hypoxia. Nystatin was added at a concentration of $160 \mu \mathrm{g} / \mathrm{ml}$. Measurements of $\mathrm{QO}_{2}$ were made in a closed, magnetically stirred, 1.6-ml thermostatted chamber using a Clark-type oxygen electrode.

Chemical assays. Total cell potassium content was measured by first layering a $0.5-\mathrm{ml}$ sample onto $0.4 \mathrm{ml}$ of a $2: 1$ mixture of dibutyldioctyl phthalate in a 1.5 -ml microcentrifuge tube, which was centrifuged for at least $10 \mathrm{~s}$. After discarding the supernatant (in samples obtained during anoxia and reoxygenation, $5-10 \%$ of the total protein was found in the supernatant) and the phthalate, the pellet was dissolved in $0.1 \mathrm{ml}$ of $14 \%$ perchloric acid, and the potassium content was measured using an atomic absorption spectrophotometer (model 460; Perkin-Elmer Corp., Norwalk, CT). Protein was measured on the same sample by the biuret method (15).

LDH was assayed according to the procedure of Bergmeyer et al. (16). Released LDH from the cells was expressed as percentage of total LDH (released LDH plus nonreleased LDH in the cells). Total LDH was measured after plasma membrane disruption by freeze thawing.

Adenine nucleotides and their breakdown products were measured using an HPLC system (Waters Associates, Milford, MA). Intracellular contents were calculated from the differences between total suspension and supernatant contents. To measure the total suspension content of the cells and the extracellular medium, $0.5-\mathrm{ml}$ samples were added to equal volumes of ice-cold, $6 \%$ perchloric acid. To measure the contents of the extracellular medium, $0.5-\mathrm{ml}$ aliquots of the suspension were first layered onto $0.4 \mathrm{ml}$ of a $2: 1$ mixture of dibutyl/dioctyl phthalate in a $1.5-\mathrm{ml}$ microcentrifuge tube, and centrifuged for at least $10 \mathrm{~s}$. The resulting supernatant was then added to an equal volume of $6 \%$ perchloric acid. In both of these types of sample, the supernatant remaining after the protein was precipitated by centrifugation was transferred to another tube, neutralized with a small volume of $2.14 \mathrm{M} \mathrm{KOH}-1.55$ $\mathrm{M} \mathrm{K}_{2} \mathrm{CO}_{3}$, and centrifuged to remove the precipitate. The supernatant was stored at $-20^{\circ} \mathrm{C}$ until assayed by HPLC. A gradient system, using $100 \mathrm{mM} \mathrm{NH}_{4} \mathrm{H}_{2} \mathrm{PO}_{4}$ (pH 5.5) and methanol, with a 5- $\mu \mathrm{m}$ Bondapak column (Waters Associates) was used to separate the nucleotides. This procedure permitted the quantitative measurement of ATP, ADP, AMP, hypoxanthine (HX), and xanthine (17).

Total GSH (reduced and oxidized; $2 \times$ ) contents were determined in the tubule suspension by the method of Griffith (18), which is based on sequential oxidation by 5,5'-dithiobis-(2-nitrobenzoic acid) and reduction by NADPH in the presence of GSH reductase. The rate of 2-nitro-5-thiobenzoic acid formation is monitored spectrophotometrically at $412 \mathrm{~nm}$. Samples were obtained from the total suspension and the supernatant as described for the nucleotide assays, and the intracellular content was determined by subtraction. Griffith (18) and other investigators, including ourselves (unpublished observations), have measured oxidized GSH content separately and found it to be $<5 \%$ of total GSH content during normoxia in the kidney. Since oxidized GSH has only been reported to increase in the presence of oxidizing agents, it was assumed that it remained a small fraction of total GSH during anoxia, when total content fell. Due to these considerations it has been assumed in this communication that the total GSH measured represents essentially only GSH.

Intracellular compartmentation of GSH. The intracellular compartmentation of GSH was determined as previously described (19, 20). Briefly, a 1-ml aliquot of the tubule suspension was placed in a 10 -ml beaker containing $4 \mathrm{ml}$ of ice-cold 3-( $N$-morpholino) propane sulfonic acid (MOPS) buffer (20 mM MOPS, 3 mM EDTA, pH 7.4) 
with or without digitonin $(0.4 \mathrm{mg} / \mathrm{ml})$ and magnetically stirred for $30 \mathrm{~s}$. An aliquot was taken and centrifuged through phthalate (dibutyl) phthalate:dioctyl phthalate, 2:1, for the determination of GSH concentrations in the medium. Aliquots were also taken for the determination of total GSH content (tubules plus medium). In the absence of digitonin there is only a small release of LDH (a cytosolic enzyme marker), GSH, and glutamate dehydrogenase (a mitochondrial enzyme marker) activity. A concentration of $0.4 \mathrm{mg} / \mathrm{ml}$ digitonin was selected because it causes the release of $\sim 50 \% \mathrm{LDH}$ but no release of glutamate dehydrogenase, as described in more detail in reference 20. Higher concentrations of digitonin result in the release of glutamate dehydrogenase activity. This fractionation procedure provided excellent separation between cytoplasmic and mitochondrial GSH, as described by Schnellmann et al. (19); therefore, the identical protocol was used in the present studies.

Materials. Collagenase (type 4) was obtained from Sigma Chemical Co. (St. Louis, MO) or Worthington Biochemical Corp. (Freehold, NJ). Digitonin, nystatin (mycostatin), and Hepes were obtained from Calbiochem-Behring Corp. (La Jolla, CA). Dextran T-40 and Ficoll were purchased from Pharmacia Fine Chemicals (Piscataway, NJ). Ouabain, reduced GSH, CYS, GLY, and Tris were obtained from Sigma Chemical Co. Diethyl maleate and BSO were purchased from Aldrich Chemical Co. (Milwaukee, WI) and Chemical Dynamic Corp. (South Plainfield, NJ), respectively. All other chemicals were reagent grade. Nystatin was dissolved in DMSO.

Statistics. The data are presented as the mean \pm SEM. Data were analyzed by analysis of variance or $t$ test. Multiple means were tested for significance using Fisher's protected least significant difference test and a $P$ value $<0.05$.

\section{Results}

Relationship between intracellular GSH content and protection of plasma membrane integrity. Under control conditions ( $\mathrm{MgCl}_{2}$ addition), $\mathrm{LDH}$ release was $\sim 4 \%$ before anoxia (not shown), increased to $42 \pm 7 \%$ after $40 \mathrm{~min}$ of anoxia (Fig. 1, top) and to $52 \pm 7 \%$ after that time in anoxia followed by $40 \mathrm{~min}$ of reoxygenation (Fig. 1, bottom). The GSH content for the control group was $5.6 \pm 0.7 \mathrm{nmol} / \mathrm{mg}$ protein before anoxia (not shown), decreasing to $2 \pm 0.5 \mathrm{nmol} / \mathrm{mg}$ protein after $40 \mathrm{~min}$ of anoxia (Fig. 2, top), and recovering to $3.8 \pm 0.6 \mathrm{nmol} / \mathrm{mg}$ protein after reoxygenation (Fig. 2, bottom). The addition of 1 $\mathrm{mM}$ GSH to the tubule suspension at the beginning of anoxia decreased $\mathrm{LDH}$ release to $14 \pm 1 \%$ after $40 \mathrm{~min}$ of anoxia (Fig. 1 , top). Since the release of $\mathrm{LDH}$ is taken as a measure of plasma membrane damage, exogenous GSH addition resulted in the protection of plasma membrane integrity. In an attempt to understand the mechanism(s) underlying this protective action, we first determined whether a higher retention of cellular GSH could occur under these conditions. This appeared to be the case, as shown in Fig. 2 (top). Since the added GSH is known to be metabolized to its three component amino acids during this anoxic period (10), two of these amino acids were tested under similar circumstances. GLY behaved identically to $\mathrm{GSH}$, providing protection from plasma membrane damage (Fig. 1, top) and retaining higher GSH levels (Fig. 2, top), whereas CYS provided only modest protection from plasma membrane damage (significantly different from both control and GSH conditions) and showed no improved GSH retention. The third amino acid component of GSH, glutamate was already present in the bathing medium (see Methods) and therefore was not tested separately. These experiments suggested that the enhanced intracellular GSH retention may be
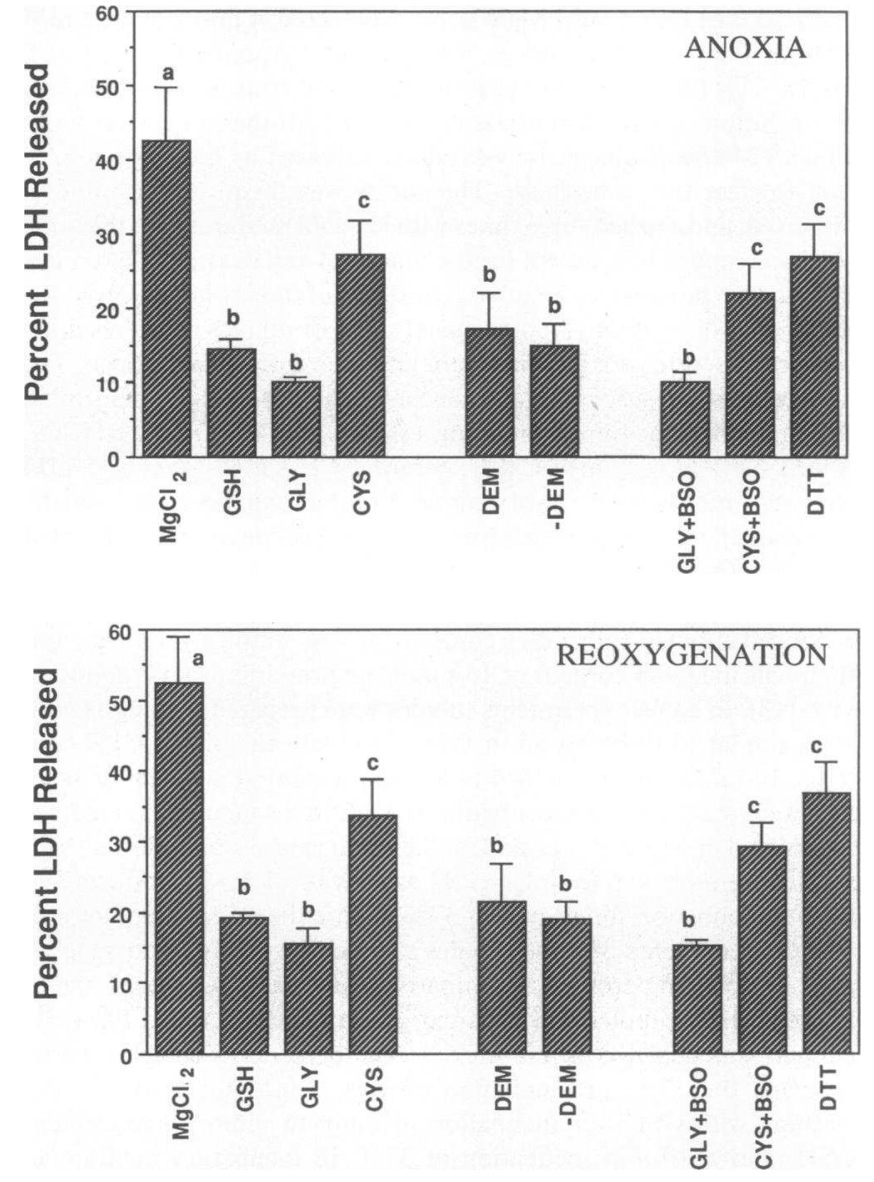

Figure 1. Percent LDH released from the tubule suspension measured either after $40 \mathrm{~min}$ of anoxia (top) or after $\mathbf{4 0} \mathrm{min}$ of anoxia and $40 \mathrm{~min}$ of reoxygenation (bottom). Number of experiments: $\mathrm{MgCl}_{2}, n=12$; all other additions, $n=4$. Additions were made as described in the text. Bars with different superscripts are significantly different from each other $(P \leq 0.05)$.

associated with the protection of plasma membrane integrity from an anoxic insult.

To test this possible role of GSH directly, a series of experiments was performed in which tubular GSH contents were both increased and decreased before anoxia. GSH contents were increased (as described in Methods) to $\sim 11 \mathrm{nmol} / \mathrm{mg}$ protein, close to the value obtained in vivo (13). As described in Methods, a paired sample was incubated for $10 \mathrm{~min}$ in 2 mM DEM to rapidly alkylate the GSH, reducing its contents to $2.5 \pm 0.1 \mathrm{nmol} / \mathrm{mg}$. Subjecting both of these groups (labeled -DEM and DEM, respectively) to $40 \mathrm{~min}$ of anoxia, elicited little $\mathrm{LDH}$ release, providing equal protection from membrane damage to that obtained by GSH and GLY (Fig. 1, top). These four groups offered identical protection despite the enormous difference in their GSH contents during anoxia, ranging from 2 to $6 \mathrm{nmol} / \mathrm{mg}$ protein (Fig. 2, top). This series of experiments brought into question the possibility that the protective effect was mediated through the intracellular GSH content. Further evidence against this possibility was provided by experiments in which GLY was added in conjunction with BSO, an inhibitor of $\gamma$-glutamyl-CYS synthetase $(1,2)$, to inhibit GSH synthesis. This maneuver was seen to decrease the GSH content during anoxia to that obtained under control conditions (Fig. 

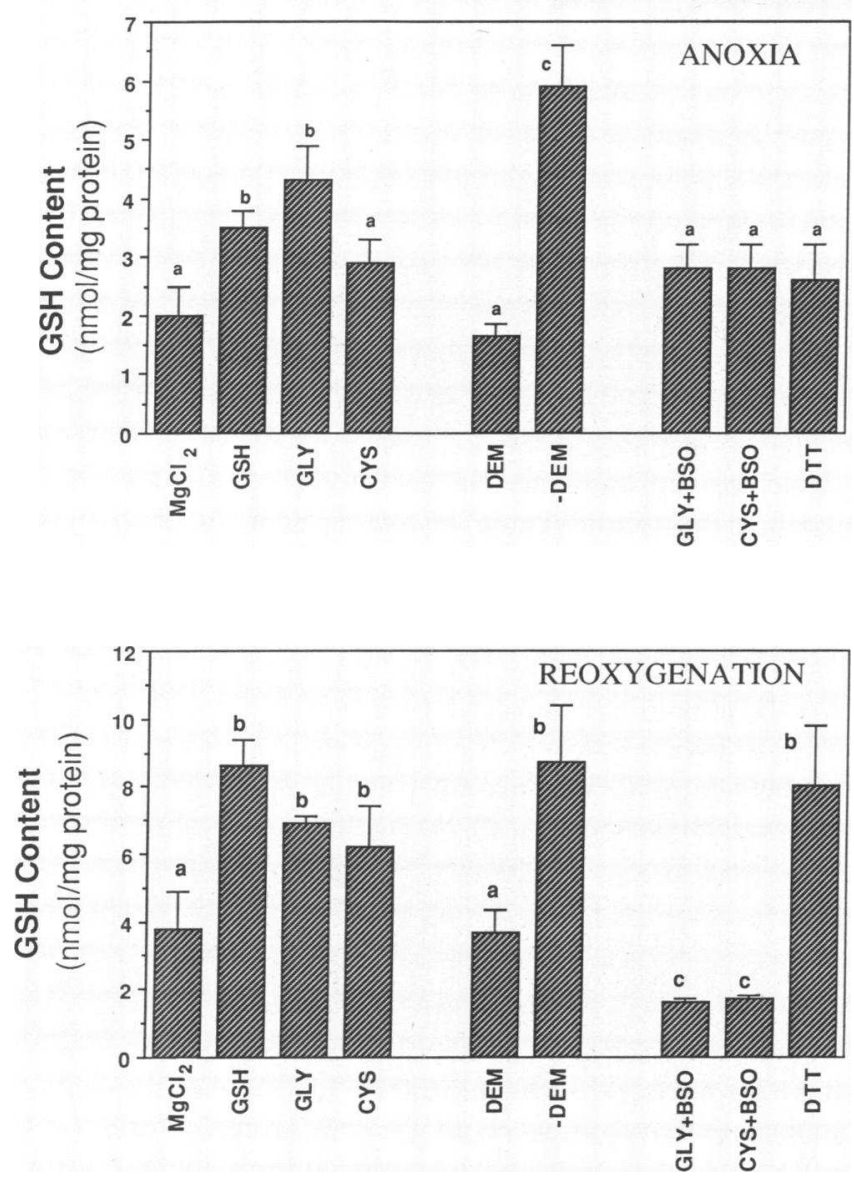

Figure 2. Intracellular GSH content of tubules subjected to $40 \mathrm{~min}$ of anoxia (top) or $40 \mathrm{~min}$ of anoxia and $40 \mathrm{~min}$ of reoxygenation (bottom). Statistics and number of experiments are as described in the legend to Fig. 1.

2, top), but it did not affect the ability of GLY to protect from plasma membrane damage (Fig. 1, top). These results strongly suggest that the cellular GSH content is unrelated to the protection from anoxic injury, indicating instead that GLY itself may be involved in such a role (see Discussion). On the other hand, the decrease in GSH content between the GLY and the GLY + BSO groups (Fig. 2, top) indicates that some GSH synthesis occurs even during anoxia.

Three other experimental conditions were tested. CYS + BSO was used as a control for the GLY + BSO experiment. This condition produced no different results from those of CYS by itself during anoxia. The last condition shown in Fig. 1 was the addition of DTT to determine whether the partial protection of the plasma membrane obtained with CYS addition was due to its reductive properties. DTT offered identical protection as CYS, suggesting that the latter agent may be acting as a reductive agent rather than a precursor of GSH. Finally, the protective effects of alanine were examined as a function of concentration, since this amino acid was not previously tested by Weinberg et al. (10). As seen in Fig. 3, $5 \mathrm{mM}$ alanine added just before anoxia provided a protective effect on LDH release equal to that of GLY.

Reoxygenation for $40 \mathrm{~min}$ led to an average of $6 \pm 2 \%$ (range, 4-10\%) additional LDH release, not significantly different from the $4 \pm 1 \%(n=4)$ released during this time period

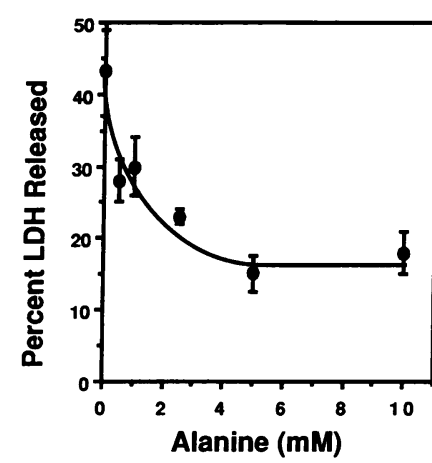

Figure 3. Percent LDH released from the tubule suspension after $40 \mathrm{~min}$ of anoxia as a function of alanine concentration. The alanine was added at the beginning of the anoxic period. Each point is an average of 4-5 experiments. by a continuously oxygenated paired control. The additional release was not significantly different among the various groups, retaining the basic pattern of $\mathrm{LDH}$ release established during anoxia (Fig. 1, bottom). This finding reiterates our previous results $(8,9)$ that most of the tubular damage is produced during anoxia in this tissue.

GSH contents recovered during reoxygenation to about their respective preoxygenated levels, except for the two groups treated with BSO, which showed a further significant decline in GSH (Fig. 2, bottom). The latter effect clearly indicates that resynthesis is required to achieve this recovery in GSH contents.

During anoxia, the control group and most other groups displayed GSH contents that decreased to $\sim 2 \mathrm{nmol} / \mathrm{mg}$ protein. Previous studies (19) demonstrated lability in cytosolic GSH content but constancy in the mitochondrial GSH content at $\sim 2 \mathrm{nmol} / \mathrm{mg}$ protein. Therefore, compartmentation studies were performed in anoxic tissue to determine whether the mitochondrial content remained relatively constant during anoxia. As shown in Table I, while the total cellular GSH content decreased from $6.4 \pm 0.4$ to $2.7 \pm 0.6 \mathrm{nmol} / \mathrm{mg}$ protein during anoxia, the mitochondrial content was $1.9 \pm 0.4 \mathrm{nmol} /$ mg protein during normoxia and was not significantly altered during anoxia. The total cellular content during anoxia was not significantly different from the mitochondrial content under either of these conditions. These results suggest that the cytosolic GSH is almost completely depleted during anoxia, whereas little change occurs in mitochondrial GSH, attesting to the integrity of mitochondria under these conditions (see Discussion).

Oxygen consumption and $K$ content. All tubules tested in these experiments displayed similar oxygen consumption patterns under preoxygenated conditions. The unstimulated

Table I. Intracellular Compartmentation of GSH during Normoxia and Anoxia

\begin{tabular}{|c|c|c|c|}
\hline \multirow[b]{2}{*}{ Experimental condition } & \multirow[b]{2}{*}{ LDH released } & \multicolumn{2}{|c|}{ GSH } \\
\hline & & Total cell & Mitochondria \\
\hline & $\%$ & \multicolumn{2}{|c|}{ nmol/mg protein } \\
\hline Normoxia & $6 \pm 1$ & $6.4 \pm 0.4$ & $1.9 \pm 0.4^{*}$ \\
\hline Anoxia & $38+5^{\ddagger}$ & $2.7 \pm 0.6^{*}$ & $1.5 \pm 0.4^{*}$ \\
\hline
\end{tabular}

* Significantly different from total cell normoxic value. All values having the same superscript are not significantly different from each other. $n=4-5$.

${ }^{\ddagger}$ Significantly different than normoxic value. 
(basal) rate averaged $27 \pm 2 \mathrm{nmol} / \mathrm{min} \cdot \mathrm{mg}$ protein, which was stimulated by $\sim 40 \%$ to $38 \pm 3 \mathrm{nmol} / \mathrm{min} \cdot$ protein upon addition of nystatin and inhibited by $\sim 50 \%$ to $14 \pm 1 \mathrm{nmol} /$ min $\cdot \mathrm{mg}$ protein in the presence of ouabain. The uncoupled rate was not significantly different from the nystatin-stimulated rate. As we have previously shown (8), $40 \mathrm{~min}$ of anoxia and reoxygenation inhibited these rates and, more specifically, the nystatin-stimulated rate only recovered to $\sim 50 \%$ of the preoxygenated value.

As seen in Table II, only GSH and GLY additions provided complete recovery of $\mathrm{QO}_{2}$. Significant but less recovery was achieved with CYS, DEM, and -DEM conditions, as well as GLY + BSO. Finally, no significant protection was obtained with CYS + BSO or DTT.

A similar recovery pattern was also observed in the $\mathrm{K}$ content. During anoxia, $\mathrm{K}$ content fell to $\sim 30 \%$ of control in all cases. $\mathrm{K}$ recovery was slightly better than $\mathrm{QO}_{2}$ recovery. Significantly better recovery of $\mathrm{K}$ content than control was observed with GSH, GLY, \pm DEM, and GLY + BSO. CYS and DTT provided no significant enhancement in recovery.

ATP content and its breakdown products. Table III shows the complex pattern of ATP breakdown during anoxia and recovery during reoxygenation, contrasting the differences among the added substances that provided protection or did not protect from anoxic damage. These studies were performed in three groups, each with its own $\mathrm{MgCl}_{2}$ control. Although the results of all control experiments were averaged in Table III, statistical comparisons were always made with the paired controls. During $\mathbf{4 0} \mathrm{min}$ of anoxia, ATP decreased by $60-80 \%$ as previously reported (9). There was no statistical difference in the anoxic ATP value among the various treatments. However, a clear difference appeared after $\mathbf{4 0} \mathrm{min}$ of reoxygenation. The percent of ATP recovery afforded by each condition is plotted in Fig. 4. Two conditions, GLY and

'Table II. Effect of GSH and Related Compounds on Nystatin-stimulated Respiration and Cellular K Content during Anoxia and Reoxygenation

\begin{tabular}{lccc}
\hline & & \multicolumn{2}{c}{$\begin{array}{c}\text { K content } \\
\text { (\% preoxygenated value) }\end{array}$} \\
\cline { 3 - 5 } Compound added & $\begin{array}{c}\text { Nystatin-stimulated } Q_{2}, \\
\text { 40-min reoxygenation } \\
\text { (\% preoxygenated value) }\end{array}$ & $\begin{array}{c}40 \text {-min } \\
\text { anoxia }\end{array}$ & $\begin{array}{c}40 \text {-min } \\
\text { reoxygenation }\end{array}$ \\
\hline Preoxygenation & $100^{*}$ & $100^{*}$ & $100^{*}$ \\
MgCl & $49 \pm 7^{\ddagger}$ & $30 \pm 2^{\ddagger}$ & $73 \pm 5^{\ddagger}$ \\
GSH & $103 \pm 4^{*}$ & $28 \pm 1^{\ddagger}$ & $103 \pm 4^{*}$ \\
GLY & $96 \pm 8^{*}$ & $33 \pm 1^{\ddagger}$ & $109 \pm 7^{*}$ \\
CYS & $80 \pm 8^{\S}$ & $30 \pm 2^{\ddagger}$ & $83 \pm 8^{\ddagger}$ \\
DEM & $78 \pm 13^{\S}$ & $38 \pm 3^{\S}$ & $96 \pm 6^{*}$ \\
-DEM & $80 \pm 15^{\S}$ & $43 \pm 2^{\S}$ & $97 \pm 2^{*}$ \\
GLY + BSO & $84 \pm 4^{\S}$ & $31 \pm 2^{\ddagger}$ & $91 \pm 2^{*}$ \\
CYS + BSO & $63 \pm 2^{\ddagger}$ & $31 \pm 1^{\ddagger}$ & $86 \pm 5^{\ddagger}$ \\
DTT & $65 \pm 5^{\ddagger}$ & $35 \pm 1^{\ddagger}$ & $77 \pm 6^{\ddagger}$ \\
\hline
\end{tabular}

Values within a column with different superscripts are significantly different from one another.

Average values before anoxia were: nystatin-stimulated respiration, $38 \pm 3 \mathrm{nmol} / \mathrm{min} \cdot \mathrm{mg}$ protein; $\mathrm{K}$ content, $340 \pm 20 \mathrm{nmol} / \mathrm{mg}$ protein; $n=4-5$.
-DEM, provided complete recovery, whereas GSH, DEM, and GLY + BSO produced significantly higher recovery than control. When alanine $(5 \mathrm{mM})$ was added the tubules displayed an identical pattern to that of GLY, decreasing ATP by $60-80 \%$ and providing complete ATP recovery after reoxygenation (data not shown).

The rest of the data in Table III are presented in an attempt to understand what conditions accompany recovery. Under preoxygenated conditions the adenine nucleotides present within the cell were mainly ATP and ADP, while the cellular AMP content was relatively small. The extracellular medium contained mainly AMP and HX, also expressed in units/nanomole per milligram protein in Table III. After $40 \mathrm{~min}$ of anoxia neither the ADP nor the AMP cellular contents seemed to change much under any of these conditions; however, significant increases in the extracellular AMP and HX contents occurred. A loss of $40-50 \%$ of total nucleotides (intracellular plus extracellular) occurred during anoxia. During reoxygenation the remaining cellular ADP and AMP, as well as the now sizeable extracellular AMP pool, were readily used for resynthesis of ATP. When comparing the various groups, some general features emerge. (a) No significant difference was observed in any of the measured variables during anoxia. The effects of the protection were only observable in these variables during reoxygenation. (b) No observable differences were seen in the ability to resynthesize ATP from cellular ADP and both cellular and extracellular AMP. (c) The differences in ATP recovery were mirrored in the ability of the tubules to increase the total nucleotide content (intracellular plus extracellular). In the groups that provided poor ATP recovery (control, DTT, $\mathrm{CYS} \pm \mathrm{BSO}$ ) the total adenine nucleotide content did not change between anoxia and reoxygenation, suggesting that the entire ATP recovery occurred from resynthesis of existing ADP and AMP. In contrast, the conditions that prompted improved recovery showed a significantly larger sum of adenine nucleotides, indicating that resynthesis of ATP could occur through other pathways under these conditions. $(d)$ Paired differences between the reoxygenated and anoxic values of HX (Fig. 5) revealed a significant increase under control conditions and the other three conditions that provided poor ATP recovery (DTT and CYS $\pm B S O$ ). On the other hand, all the conditions that promoted better ATP recovery than control showed either no change or a decrease in $\mathrm{HX}$ content.

\section{Discussion}

As we have previously demonstrated in rabbit tubules subjected to anoxia and reoxygenation $(8,9)$, most of the cellular damage occurs during anoxia. Furthermore, protection of the plasma membrane by exogenous addition of adenine nucleotides or adenosine leads to complete recovery during reoxygenation $(8,9)$. The present results extend these findings by demonstrating that GSH or GLY added during anoxia protect during that time period, and this protection is also retained during reoxygenation. Similar results were recently reported by Weinberg et al. (10), who concluded that extracellular GLY was the cytoprotective agent in both of these cases, since GSH was catabolized into its component amino acids during this time period. These investigators did not measure cellular GSH content, leaving open the possibility that this variable may 
Table III. Adenine Nucleotide and HX Contents during Normoxia, Anoxia, and Reoxygenation

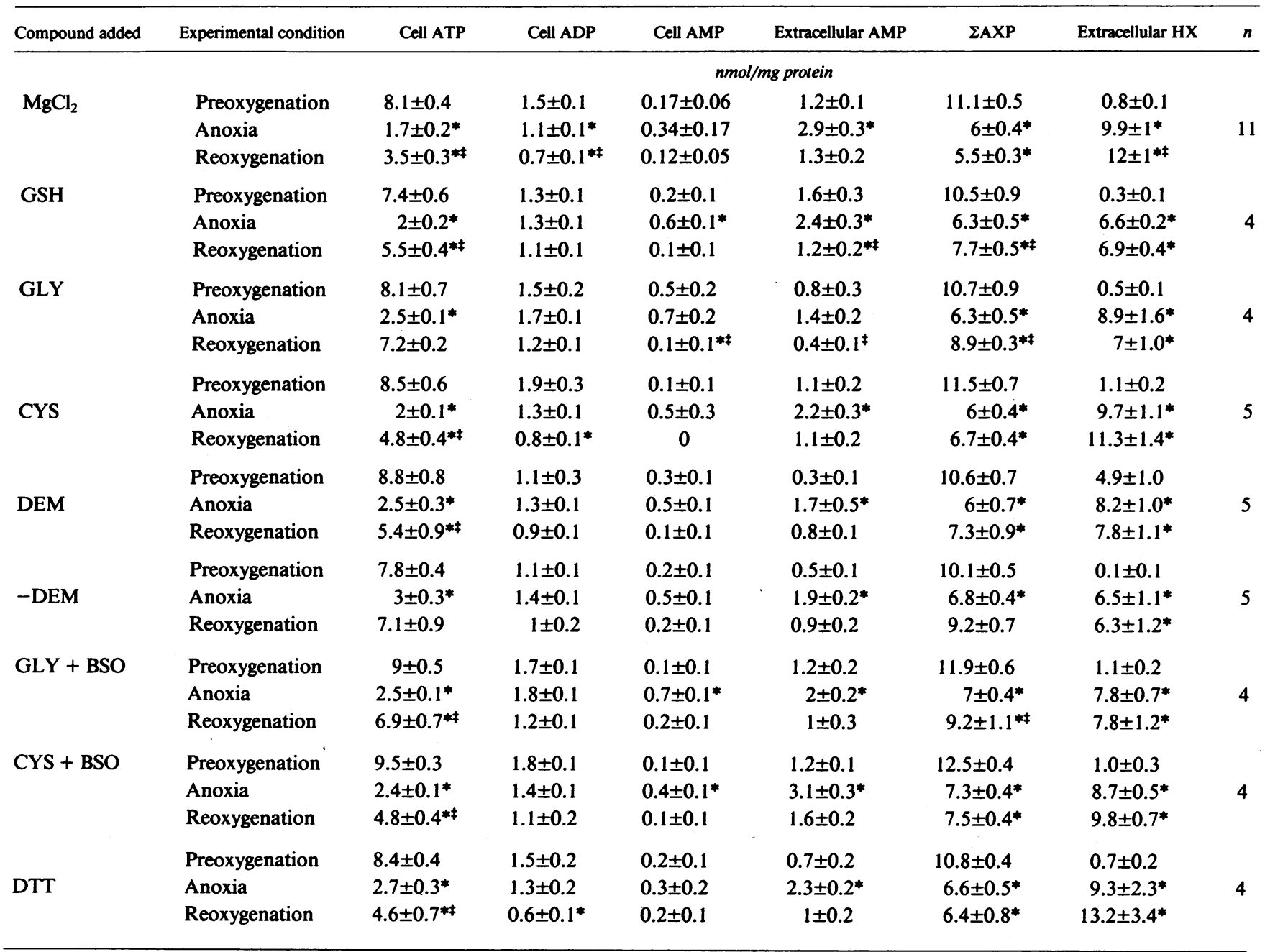

Values within a column with different superscripts are significantly different from one another. Normoxic time controls $(n=5)$ are not significantly different from $\mathrm{MgCl}_{2}$ preoxygenation values after 60 or 100 min of continuous oxygenation.

mediate the anoxic cytoprotective action. ${ }^{3}$ The present results with GSH and GLY did indeed show an elevated GSH content during anoxia in both cases (Fig. 2, top). Further experiments designed to enhance GSH contents (-DEM condition) before anoxia produced similar protection to those with added GSH or GLY at the beginning of anoxia. However, alkylation of most cellular GSH with DEM did not affect this protective action. The conclusion from these experiments is that cellular GSH is not itself cytoprotective, but that a GSH metabolite that is still available after alkylation may mediate that function. DEM reacts with the sulfur of the cysteinyl group (21), and this leaves the rest of the molecule still available for enzymatic degradation to GLY and glutamate (2). Since exoge-

3. A paper from this group was published since the submission of the present manuscript, in which cellular GSH contents were measured in proximal tubules subjected to anoxia (36). They also found increased GSH levels in tubules to which exogenous GLY or GSH was added, but eliminating this increment in GSH did not affect the protection from anoxic damage. These results are in complete agreement with ours. nously added GLY is cytoprotective, it appears reasonable to conclude that endogenously produced GLY would also offer protection. Therefore, these experiments reveal a possibly novel mechanism of cytoprotective action for intracellular GSH, which occurs through the production of GLY during anoxia. Cells with high initial GSH content would be relatively more protected during anoxia than cells with lower GSH content due to the ability of the former to produce more GLY than the latter.

The protective action of GLY is presumably at or near the plasma membrane, where its concentration would be difficult to measure. Weinberg et al. (10) found the $K_{\mathrm{m}}$ for protection by exogenously added GLY to be $0.3-0.5 \mathrm{mM}$. The GSH content of the -DEM group decreased by $\sim 5 \mathrm{nmol} / \mathrm{mg}$ protein during anoxia (Fig. 2, top), corresponding to $25 \mathrm{nmol} / \mathrm{ml}$ of suspension. Complete breakdown of this GSH would raise the concentration of GLY in the solution by $0.025 \mathrm{mM}$, about one-tenth of that needed for protection from exogenously added GLY. However, it is possible that diffusional gradients for GLY are such that its concentration at the site(s) of cytoprotective action is comparable under both of these conditions. 


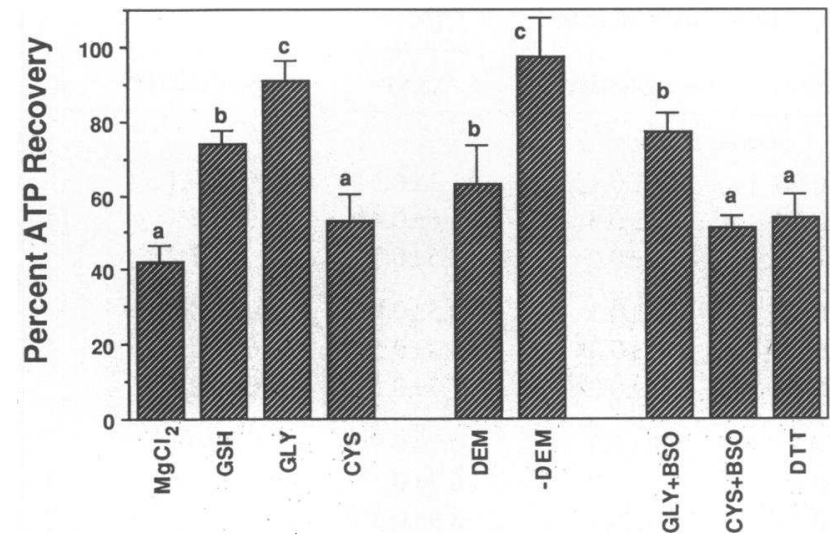

Figure 4. Percent ATP recovery of tubules subjected to $40 \mathrm{~min}$ of anoxia and $\mathbf{4 0} \mathrm{min}$ of reoxygenation. Statistics and number of experiments are as described in the legend to Fig. 1.

The nature of the protective mechanism is presently unknown, and it is unclear whether it involves the metabolism of GLY (10). Another neutral amino acid, alanine, provided complete protection during anoxia (Fig. 3), whereas other neutral amino acids such as phenylalanine (Mandel, L. J., unpublished results) and serine (10) were ineffective. ${ }^{4}$ It may be speculated that this specificity points to a possible role of these amino acids in the inhibition of specific phospholipases or proteases that affect cytoskeletal-plasma membrane interactions, leading to membrane disruption. The present experiments do not show any evidence that the protection elicited by GLY or alanine involves the ability to produce ATP anaerobically or to inhibit the rate of ATP or AMP hydrolysis during anoxia. This contrasts with preliminary results of Garza et al. (22), where alanine addition ( $5 \mathrm{mM}$ ) during anoxia caused a slight increase in ATP content, presumably through anaerobic glycolysis to succinate. The nature of the difference between these results and ours is unclear. Any ATP produced anaerobically would be expected to be protective, as documented in the perfused kidney (23), thick ascending limbs (24), proximal straight segments (25), and cells in culture (26). If convoluted proximal tubules could be made to produce ATP anaerobically, this could provide some protection. However, in our laboratory neither our previous experiments with adenine nucleotide addition (9) nor the present experiments show increased ATP levels during anoxia. The same result was obtained by Weinberg et al. $(10,27)$ with adenine nucleotides, GLY, and GSH. Therefore, it appears that in this segment the main protective mechanism during anoxia resides elsewhere, as discussed earlier.

The protective action of GSH during anoxia needs to be taken into consideration when in vivo experiments are performed subjecting the kidney to ischemia and reoxygenation. Under these conditions protection by GSH is usually attributed to its ability to react with oxygen free radicals presumably produced during reoxygenation (4-7). The present experi-

4. A very recent communication by Silva et al. (37), which appeared after submission of this manuscript, reported protection of thick ascending limbs from hypoxic damage by GLY and alanine. Therefore, the protective effects of these amino acids may not be restricted to proximal renal segments.

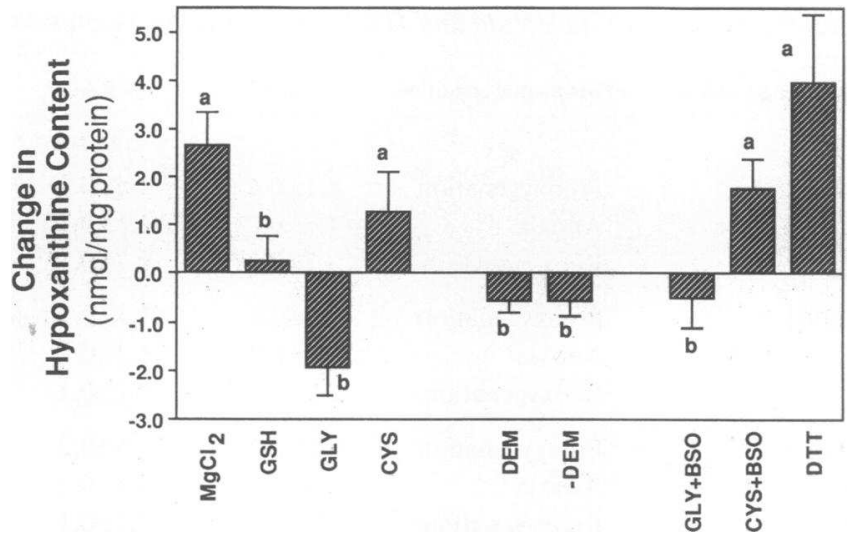

Figure 5. Paired differences in the HX content found after 40 min of reoxygenation minus that found after $\mathbf{4 0} \mathrm{min}$ of anoxia. These differences were calculated for each individual experiment and were subsequently averaged for each experimental condition. Statistics and number of experiments are as described in the legend to Fig. 1.

ments suggest an alternative interpretation, namely, that GSH may be protective during anoxia and this protection prevails during reoxygenation.

The experiments with BSO show that some GSH resynthesis occurs even during anoxia, indicating on the one hand that this process must have a low ATP requirement, and on the other hand that this process appears to be ATP limited during anoxia, since reoxygenation leads to complete recovery of GSH contents. It is noteworthy that most of the GSH depletion that occurs during anoxia originates from the cytosolic compartment. This result suggests that the relative mitochondrial impermeability to $\operatorname{GSH}(1,2)$ observed during normoxia is retained during anoxia. Recent observations from various laboratories $(28,29)$ have suggested that mitochondrial permeability properties are maintained or even tightened during anoxia, and the present results also confirm this observation.

The partial protection offered by DTT and CYS during anoxia was somewhat surprising, since reducing agents would not be expected to protect during anoxia. On the other hand, no protection by these reducing agents is evident in K or ATP contents during reoxygenation, confirming the lack of reoxygenation-induced damage in this preparation. Nystatin-stimulated respiration appeared to be somewhat protected by CYS but not by DTT addition or CYS + BSO. The former may just be an aberrant observation, since it is not reflected in any of the other variables.

In general, the oxygen consumption as well as the $K$ and ATP contents reflected a pattern closely related to that of the LDH release. Conditions that inhibited LDH release during anoxia led to better recovery of these variables during reoxygenation. Cellular $\mathrm{K}$ contents were slightly higher during anoxia in the DEM and -DEM conditions but otherwise were not different from each other. The recovery of $\mathrm{K}$ content was generally higher than the recoveries of either $\mathrm{QO}_{2}$ or ATP content. A partial explanation for this difference may reside in the method used to measure $\mathrm{K}$ content, which involves centrifugation through a layer of oil to minimize extracellular $\mathrm{K}$ (see Methods). The cellular $\mathrm{K}$ contents are measured on the tubules below the oil layer, and these may comprise only relatively healthy tubules, since damaged tubules may be trapped 
above the oil layer. On the other hand, ATP contents and $\mathrm{QO}_{2}$ measurements are made on the total suspension.

The results in Table III are consistent with previous results $(9,30,31)$ showing that resynthesis of ATP occurs in two steps. The initial step involves resynthesis from existing adenine nucleotide precursors, which seems to occur rapidly after reoxygenation (30), followed by a slower recovery over the next few hours. Since the sum of total adenine nucleotides after $\mathbf{4 0} \mathrm{min}$ of anoxia was the same under all of the tested conditions, it may be inferred that the initial recovery of ATP may not have been different either. The slower recovery, which depends on ATP resynthesis from sources other than ADP and AMP, appears to have been better preserved by GSH, GLY, and DEM. Since this recovery was associated with better retention of LDH, it may be assumed that the enzymes necessary for such ATP resynthesis were retained too.

Various possible pathways could be involved, including purine salvage from $\mathrm{HX}(32,33)$. The lack of change in $\mathrm{HX}$ levels between anoxia and reoxygenation in the conditions which lead to better ATP recovery (Fig. 5) may argue against the latter possibility; however, it is possible that the lack of net change may obscure a concurrent continuing release of $\mathrm{HX}$ from damaged cells and HX resynthesis into ATP in healthy cells. Further studies are needed to clarify this point.

We $(9)$ and others $(34,35)$ have shown that a steady decrease in total adenine nucleotide pool occurs as a function of time in anoxia. In the rabbit, the main breakdown product is HX. The data in Table III clearly show that another source of $\mathrm{HX}$ exists, since the increase in $\mathrm{HX}$ is much larger than the decrease in the sum of adenine nucleotides during anoxia. The most probable sources are NAD and/or RNA, and some data exist to document the decrease in both of these compounds during anoxia (8). The contrast obtained during reoxygenation between the further increase in $\mathrm{HX}$ content with $\mathrm{MgCl}_{2}$ addition (control) against the decrease or lack of change in this value under protective conditions is remarkable. This suggests that the direction of change in $\mathrm{HX}$ levels during reoxygenation may be predictive of reversibility of cellular damage.

In conclusion, tubules were protected from anoxic damage by either endogenous or exogenous GSH. The protection occurred during anoxia and the protective agent was GLY, one of the constituent amino acids of GSH. Plasma membrane damage during anoxia was inhibited by exogenous addition of GLY or alanine through as yet unknown mechanisms.

\section{Acknowledgments}

The authors would like to thank Mrs. Jinny Shelton for typing the manuscript.

This work was supported by National Institutes of Health grant DK-26816.

\section{References}

1. Meister, A., and M. E. Anderson. 1983. Glutathione. Annu. Rev. Biochem. 52:711-760.

2. Meister, A. 1988. Glutathione metabolism and its selective modification. J. Biol. Chem. 263:17205-17208.

3. Jennische, E. 1984. Possible influence of glutathione on postischemic liver injury. Acta Pathol. Microbiol. Immunol. Scand. Sect. A Pathol. 92:55-64.

4. Paller, M. S. 1986. Hypothyroidism protects against free radical damage in ischemic acute renal failure. Kidney Int. 29:1 162-1166.

5. McCoy, R. N., K. E. Hill, M. A. Ayon, J. H. Stein, and R. F.
Burk. 1988. Oxidant stress following renal ischemia: changes in the glutathione redox ratio. Kidney Int. 33:812-817.

6. Paller, M. S. 1988. Renal work, glutathione and susceptibility to free radical-mediated postischemic injury. Kidney Int. 33:843-849.

7. Scaduto, R. C., Jr., V. H. Gattone II, L. W. Grotyohann, J. Wertz, and L. F. Martin. 1988. Effect of an altered glutathione content on renal ischemic injury. Am. J. Physiol. 255:F911-F921.

8. Takano, T., S. P. Soltoff, S. Murdaugh, and L. J. Mandel. 1985. Intracellular respiratory dysfunction and cell injury in short-term anoxia of rabbit renal proximal tubules. J. Clin. Invest. 76:2377-2384.

9. Mandel, L. J., T. Takano, S. P. Soltoff, and S. Murdaugh. 1988. Mechanisms whereby exogenous adenine nucleotides improve rabbit renal proximal function during and after anoxia. J. Clin. Invest. 81:1255-1264.

10. Weinberg, J. M., J. A. David, M. Abarzua, and T. Rajan. 1987. Cytoprotective effects of glycine and glutathione against hypoxic injury to renal tubules. J. Clin. Invest. 80:1446-1454.

11. Balaban, R. S., S. Soltoff, J. M. Storey, and L. J. Mandel. 1980. Improved renal cortical tubule suspension: spectrophotometric study of $\mathrm{O}_{2}$ delivery. Am. J. Physiol. 238:F50-F59.

12. Soltoff, S. P., and L. J. Mandel. 1984. Active ion transport in the renal proximal tubule. I. Transport and metabolic studies. J. Gen. Physiol. 84:601-622.

13. Schnellmann, R. G., E. A. Lock, and L. J. Mandel. 1987. A mechanism of S-(1,2,3,4,4-pentachloro-1,3-butadienyl)-L-cysteine (PCBC) toxicity to rabbit renal proximal tubules. Toxicol. Appl. Pharmacol. 90:513-521.

14. Harris, S. I., R. S. Balaban, L. Barrett, and L. J. Mandel. 1981. Mitochondrial respiratory capacity and $\mathrm{Na}^{+}$- and $\mathrm{K}^{+}$-dependent adenosine triphosphatase-mediated ion transport in the intact renal cell. $J$. Biol. Chem. 256:10319-10328.

15. Layne, E. 1957. Spectrophotometric and turbidimetric methods for measuring proteins. Methods Enzymol. 3:447-454.

16. Bergmeyer, H. U., E. Bernt, and B. Hess. 1963. Lactic dehydrogenase. In Methods of Enzymatic Analysis. H. U. Bergmeyer, editor. Academic Press, Inc., New York. 736-743.

17. Hull-Ryde, E. A., R. G. Cummings, and J. E. Lowe. 1983. Improved method for high energy nucleotide analysis of canine cardiac muscle using reversed-phase high-performance liquid chromatography. J. Chromatogr. 275:411-417.

18. Griffith, O. W. 1980. Determination of glutathione and glutathione disulfide using glutathione reductase and 2-vinylpyridine. Anal. Biochem. 106:207-212.

19. Schnellmann, R. G., S. M. Gilchrist, and L. J. Mandel. 1988. Intracellular distribution and depletion of glutathione in rabbit renal proximal tubules. Kidney Int. 34:229-233.

20. Schnellmann, R. G., and L. J. Mandel. 1985. Intracellular distribution of glutathione in rabbit renal proximal tubules. Biochem. Biophys. Res. Commun. 133:1001-1005.

21. Chausseaud, L. F. 1979. The role of glutathione and glutathione S-transferases in the metabolism of chemical carcinogens and other electrophilic agents. Adv. Cancer Res. 29:175-274.

22. Garza, R., J. Ortega, J. Stein, and M. Venkatachalam. 1989. Strategies to increase ATP generation in anoxic proximal tubules. Kidney Int. 35:407. (Abstr.)

23. Gronow, G. H., and J. J. Cohen. 1984. Substrate support for renal functions during hypoxia in the perfused rat kidney. Am. J. Physiol. 247:F838-F843.

24. Chamberlin, M. E., and L. J. Mandel. 1987. $\mathrm{Na}^{+}-\mathrm{K}^{+}-A$ TPase activity in medullary thick ascending limb during short-term anoxia. Am. J. Physiol. 252:F838-F843.

25. Ruegg, C. E., and L. J. Mandel. 1989. Bulk separation of rabbit PCT and PST: substrate-dependent metabolic differences. Kidney Int. 35:416. (Abstr.)

26. Venkatachalam, M. A., Y. J. Patel, J. I. Kreisberg, and J. M. Weinberg. 1988. Energy thresholds that determine membrane integrity and injury in a renal epithelial cell line $\left(\mathrm{LLC}_{\mathrm{P}} \mathrm{PK}_{1}\right) . \mathrm{J}$. Clin. Invest. $81: 745-758$. 
27. Weinberg, J. M., J. A. Davis, A. Lawton, and M. Abarzua. 1988. Modulation of cell nucleotide levels of isolated rabbit kidney tubules. Am. J. Physiol. 23:F311-F322.

28. Lemasters, J. J., J. DiGuiseppi, A. L. Nieminen, and B. Herman. 1987. Blebbing, free $\mathrm{Ca}^{+}$and mitochondrial membrane potential preceding cell death in hepatocytes. Nature (Lond.). 325:78-81.

29. Anderson, B. S., T. Y. Aw, and D. P. Jones. 1987. Mitochondrial transmembrane potential and $\mathrm{pH}$ gradient during anoxia. Am. J. Physiol. 252:C349-C355.

30. Stromski, M. E., K. Cooper, G. Thulin, K. M. Gaudio, N. J. Siegel, and R. G. Shulman. 1986. Chemical and functional correlates of postischemic renal ATP levels. Proc. Natl. Acad. Sci. USA. 83:6142-6145.

31. Stromski, M. E., A. v. Waarde, M. J. Avison, G. Thulin, K. M. Gaudio, M. Kashgarian, R. G. Shulman, and N. J. Siegel. 1988. Metabolic and functional consequences of inhibiting adenosine deaminase during renal ischemia in rats. J. Clin. Invest. 82:1694-1699.

32. Fox, I. H. 1978. Degradation of purine nucleotides. Handb. Exp. Pharmacol. 51:94-124.
33. Gerlach, E., P. Marko, H. G. Zimmer, I. Pechan, and Ch. Trendelenburg. 1970. Different response to adenine nucleotide synthesis do novo in kidney and brain during aerobic recovery from anoxia and ischemia. Experientia (Basel). 27:876-878.

34. Busch, E. W., I. M. Von Borcke, and B. Martinez. 1968. Abbauwege und Abbaumuster der Purinnucleotide in Herz-, Leber-, und Nierengewebe von Kaninchen nach Kreislauf-stillstand. Biochim. Biophys. Acta. 166:546-556.

35. Buhl, M. R. 1979. The predictive value of 5'-adenine nucleotide depletion and replenishment in ischaemic rabbit kidney tissue. Int. Urol. Nephrol. 11:325-333.

36. Weinberg, J. M., J. A. Davis, M. Abarzua, and Tal Kiani. 1989. Relationship between cell adenosine triphosphate and glutathione content and protection by glycine against hypoxic proximal tubule cell injury. J. Lab. Clin. Med. 113:612-622.

37. Silva, P., K. C. Spokes, S. Rosen, and F. H. Epstein. 1989. Glycine protects the thick ascending limb from hypoxic damage. Clin. Res. 37:585A. (Abstr.) 